\title{
Dynamique de Colonisation des Zones Rudérales d'un Massif Forestier Urbain par les Espèces Végétales Exotiques Envahissantes : Cas du Parc National du Banco (Côte d'Ivoire)
}

\author{
Akaffou Sopie Elvire Vanessa, \\ Mévanly Ouattara, \\ Gouli Gnanazan Zinsi Roseline, \\ Tiébré Marie-Solange, \\ Laboratoire de Botanique, UFR Biosciences, \\ Université Félix Houphouët-Boigny, Abidjan, Côte d'Ivoire
}

Doi:10.19044/esj.2019.v15n21p240 URL:http://dx.doi.org/10.19044/esj.2019.v15n21p240

Résumé

Les zones rudérales constituent des points de départ pour l'envahissement des écosystèmes naturels par les plantes invasives. Dans cette étude, il s'agit inventorier toutes les plantes invasives présentes dans le PNB, de mettre en évidence leur distribution spatiale et de suivre leur évolution d'occupation du sol. Les données floristiques ont été recueillies d'abord par un inventaire itinérant afin de recenser et identifier toutes les plantes invasives. A l'issue de cet inventaire six zones de forte présence de plantes invasives ont été sélectionnées : sous les lignes de haute tension, les abords de chemin, l'arboretum, les campements (campement détruit et campement actuel), la périphérie du parc et la portion de la forêt d'Anguédédou qui représente la zone non rudérale occupée par les espèces invasives. Par la suite, des relevés phytosociologiques réalisés au sein de 203 parcelles permanentes de $50 \mathrm{~m}^{2}$. A l'intérieur de chaque parcelle, toutes les espèces invasives ont été recensées., dénombrées et leurs surfaces d'occupation du sol ont été mesurées consécutives, de 2016 à 2018. 22 espèces dénombrées dont une invasive avérée et 21 potentiellement invasives repartie sur l'ensemble du parc. Chromolaena odorata et Hopea odorara constituent une menace réelle pour ce parc. Les campements, la périphérie du parc sont les endroits les plus envahis. La dynamique spatio-temporelle des espèces invasives montre qu'elles occupent 12,01 ha des zones rudérales du parc et 126,84 ha soit 3,68 $\%$ en prenant en compte de la superficie plantée de Hopea odorata. Ces résultats permettent d'anticiper et coordonner des actions en vue d'éviter l'envahissement à grande échelle du parc. 
Mots-clés : Zones rudérales, Plantes invasives, Dynamique spatio-temporelle, Parc National du Banco

\title{
Dynamics of Colonization of the Ruderal Zones of an Urban Forest by Invasive Alien Plant Species: Case of the Banco National Park (Côte d'Ivoire)
}

\author{
Akaffou Sopie Elvire Vanessa, \\ Mévanly Ouattara, \\ Gouli Gnanazan Zinsi Roseline, \\ Tiébré Marie-Solange, \\ Laboratoire de Botanique, UFR Biosciences, \\ Université Félix Houphouët-Boigny, Abidjan, Côte d'Ivoire
}

\begin{abstract}
The ruderal areas are starting points for invasion of natural ecosystems by invasive exotic plant. In this study we have inventoried all the invasive plants present in the PNB, highlighted their spatial distribution and followed their evolution of occupation of the soil. The floristic data were first collected by itinerant inventory in the study area. At the end of this inventory, six zones of strong presence of invasive plants were selected: under of high voltage lines, the roadside, the arboretum, the encampments (destroyed encampment and current encampment), the periphery of the park and the portion of Anguédédou forest attached to park, which represents the not ruderal zone occupied by invasive species. Thereafter that of phytosociological records carried inside 203 permanent plots of $50 \mathrm{~m}^{2}$. Inside each plot, all invasive species were inventory, counted, and their area of soil occupation was measured consecutive, from 2016 to 2018. 22 species counted, one of which proven invasive and 21 potentially invasive distributed throughout the park. Chromolaena odorata and Hopea odorara constitute a real threat to this park. The encampments, the periphery of the park are the most invaded places in the park. The spatio-temporal dynamics of the species species occupy 12,01 ha of the ruderal areas of the park and 126,84 ha taking into account the planted area of Hopea odorata is $3,68 \%$ of the total surface of the park. These results will make it possible to anticipate and of coordinate actions in order to avoid the large-scale invasion of the park
\end{abstract}


Keywords: Ruderals areas, Invasive exotic plant, Spatio-temporal dynamics, Banco National Park

\section{Introduction}

Les zones rudérales se définissent comme des milieux influencés par l'homme, à savoir les bords de routes, de chemins, de pistes, les bords des massifs forestiers, les zones piétinées, les friches, les voies ferrées, les terrains vagues, les voies de fil électriques, les décharges, les décombres, les dépotoirs, etc. (Nyakabwa, 1981 ; Brun, 2007 ; Uchida et al., 2014). Ces zones renferment des plantes utiles qui peuvent avoir une valeur nutritive et médicinale pour l'homme ou fourragère pour les animaux domestiques (Nyakabwa, 1981). Plusieurs études ont montré que les zones rudérales constituent des points de départ pour l'envahissement des écosystèmes naturels par les plantes invasives (Tyser \& Worley, 1992 ; Tiébré et al., 2008). Ces dernières se définissent comme des espèces exotiques (ou étrangères) dont l'introduction, délibérée ou accidentelle par l'homme, l'établissement et la dissémination menacent la biodiversité, les écosystèmes, les habitats et les communautés (UICN, 2000 ; Tiébré et al., 2000 ; Levine, 2008 ; Dumitraşcu et al., 2012 ; Hulme, 2016 ; Simberloff, 2016). Les plantes invasives constituent la deuxième cause directe de perte de la biodiversité dans le monde (Vitousek et al., 1996 ; UICN, 2000 ; MEDD, 2004 ; MEA, 2005). Elles engendrent de nombreux impacts négatifs sur le plan écologique, environnemental, social et économique (Pimentel et al., 2000 ; Marck et al., 2000 ; McNeely et al., 2001 ; Selvi et al., 2016). Sur le plan économique, le coût des espèces invasives est estimé à 150 milliards d'euros par an aux EtatsUnis. Dans l'Union Européenne, ce coût pourrait atteindre 10 milliards d'euros, sachant que l'impact environnemental est inconnu dans $90 \%$ des cas (Hulme et al., 2009).

C'est fort de ce constat que, de nombreux programmes de recherches ont été lancés dans plusieurs pays sur l'inventaire des espèces invasives ou Espèces Exotiques Envahissantes, nommées communément EEE (Roux, 2011 ; Dortel \& Geslin, 2016). En effet, Roux (2011) évoque qu'il existe une complexité au niveau des paramètres à prendre en compte pour définir clairement une espèce invasive. Il a retenu 4 critères pour définir une espèce invasive : l'introduction de l'espèce dans une région à partir de 1500 après J.C ; l'impact avéré de sa présence ; le constat d'une reproduction effective et la notion de prolifération en terme du niveau d'impact occasionné par l'espèce. Grâce à ces critères, il a été possible d'élaborer une proposition de liste d'espèces invasives en Sarthe, en Bretagne et en Pays de Loire. Plusieurs espèces ont été écartées de la liste car il était difficile d'établir facilement leur statut. Dortel \& Geslin (2016), Quere \& Geslin (2016) ont aussi défini des 
critères pour l'évaluation du statut d'invasibilité des espèces exotiques. Selon ces auteurs, il est important de tenir compte : du statut d'indigénat de la plante ; du caractère envahissant ou de la tendance à montrer un caractère envahissant dans le territoire considéré ; du caractère envahissant dans un territoire géographiquement proche ou dans d'autres régions du monde à climat proche ; du caractère envahissant en milieu naturel ou semi naturel ou uniquement en milieu fortement anthropisé; de l'impact de la colonisation de l'espèce sur la biodiversité, de la santé humaine et des activités économiques. Ces critères ont abouti à une classification des plantes invasives en : invasives avérées (IA) ; invasives potentielles (IP) ; Plantes à surveiller (AS) et non invasives (NI). La réalisation de l'inventaire des espèces invasives est un outil indispensable pour programmer et optimiser la conduite des opérations de contrôle engagées par les gestionnaires ainsi que pour évaluer l'emprise annuelle, tant géographique qu'en intensité de l'invasion des plantes. La mise à jour annuelle de cet inventaire permet de suivre l'évolution rapide des colonisations, d'apporter une compréhension globale des dynamiques en place et d'élaborer des stratégies de gestion durable (Gourié, 2004 ; Thiombiano et al., 2009 ; Haury \& Damien, 2012 ; Guyé et al., 2015 ; Nzengue et al., 2016 ; Abdou et al., 2017).

Les aires protégées sont aussi de plus en plus exposées à la prolifération des espèces invasives (Carrière et al., 2008 ; Foxcroft et al., 2013 ; Foxcroft et al., 2016, Nzengue et al., 2016). Le Parc National du Banco (PNB) est un massif forestier relique du littoral de Côte d'Ivoire, caractérisé par une diversité de micro-habitats naturels et anthropisés (Sako \& Beltrando, 2014). Cette biodiversité lui confère une importante valeur pour la conservation (Lauginie, 2007). Des études récentes montrent que cette forêt urbaine est en proie à de nombreuses pressions anthropiques, notamment l'urbanisation et l'agriculture périphérique (Sako et al., 2013 ; Sako \& Beltrando, 2014 ; Tia \& Dago, 2015 ; Akoue et al., 2017). Ces facteurs concourent à l'accroissement des zones rudérales et favorisent ainsi leur colonisation par les plantes invasives. En effet, divers travaux ont montré que le PNB renferme de nombreuses espèces invasives qui ont pris des proportions inquiétantes (Tiébré et al., 2014 ; 2015 ; Tiébré \& Gouli Gnanazan, 2018). Par conséquent, il devient urgent d'inventorier les espèces exotiques envahissantes présentes dans le parc et de suivre leur dynamique de colonisation afin de préserver la biodiversité au sein de ce patrimoine forestier. La présente étude s'est fixée comme objectif général de contribuer à une meilleure connaissance des espèces exotiques envahissantes du PNB. Il s'est agi plus spécifiquement de : (1) recenser toutes les plantes invasives présentes dans le parc, (2) mettre en évidence la distribution spatiale de ces espèces et (3) suivre l'évolution de l'occupation du sol de ces plantes invasives. 


\section{Matériel et méthodes}

\section{Site d'étude}

L'étude s'est déroulée dans le Parc National du Banco (PNB) localisé dans le district autonome d'Abidjan entre $5^{\circ} 21^{\prime}-5^{\circ} 25^{\prime} \mathrm{N}$ et $4^{\circ} 11^{\prime}-4^{\circ} 5^{\prime} \mathrm{O}$ (figure 1). C'est une relique de forêt littorale primaire psammohygrophile de 3474 ha. Le climat est de type tropical (Peel et al., 2007) caractérisé par quatre saisons : une petite saison sèche d'août à septembre et une grande de décembre à mars, d'une grande saison des pluies d'avril à juillet et d'une petite d'octobre à novembre (Eldin, 1971). Les précipitations moyennes annuelles enregistrées par la station météorologique SODEXAM pour la période de 2000 à 2009 sont de $1733 \mathrm{~mm}$. La température moyenne est de $27,4^{\circ} \mathrm{C}$ avec une amplitude de $4,3^{\circ}$ C. Le sol du Parc National du Banco de type ferrasol (FAO, 2006) est caractérisé par un sol sableux, ferralitique, fortement désaturé (Perraud, 1971). Selon De Koning (1983) et Lauginie (1996), le PNB est une forêt sempervirente. Les arbres dominants des strates supérieures sont Turraeanthus africanus (Welw. ex C. DC.) Pellegr., Synsepalum afzelii (Engl.) T. D. Penn., Berlinia confusa Hoyle, Blighia welwitschii (Hiern) Radlk., Coula edulis Baill., Dacryodes klaineana (Pierre) H.J. Lam, Lophira alata Banks ex Gaertn.f., Petersianthus macrocarpus (P. Beauv.) Liben et Piptadeniastrum africanum (P. Beauv.) Liben.
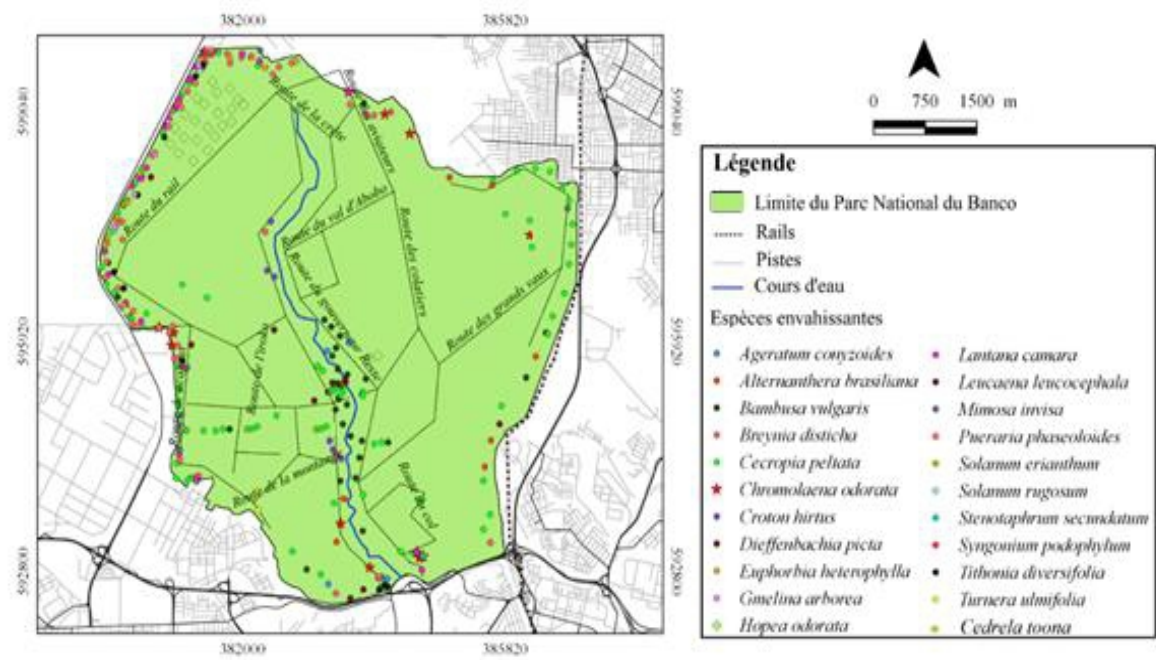

Figure 1 : Carte de la localisation et de la distribution des espèces invasives du Parc National du Banco

\section{Collecte des données}

A l'issue des travaux de prospection réalisés dans le Parc National du Banco, six biotopes contenant des plantes invasives ont été sélectionnées : il s'agit de la zone d'emprise des lignes de haute tension, les abords de chemin, 
l'arboretum, les campements (campement détruit et campement actuel), la périphérie du parc et la portion de forêt d'Anguédédou rattachée au Parc National du Banco qui représente la zone non rudérale occupée par les espèces invasives.

Les données ont été recueillies selon deux méthodes de relevés : la première a consisté en un inventaire itinérant (N'Guessan \& Kassi, 2018 ; Ouattara et al., 2018). Il s'agit de parcourir les biotopes dans toutes les directions afin de recenser et identifier les espèces végétales invasives et les localiser à l'aide d'un GPS. La surface d'occupation de ces plantes invasives a été mesurée. Ensuite, le contour des peuplements a été levé avec le GPS pour déterminer la surface d'occurrence des espèces. Le recouvrement de chaque espèce invasive a été évalué. La seconde méthode utilisée est celle des relevés de surface. Elle a été réalisée à l'intérieur des placettes de $50 \mathrm{~m}^{2}(5 \mathrm{~m} \mathrm{x} 10 \mathrm{~m})$. Toutes les espèces végétales ont été dénombrées et identifiées. Les fréquences relatives ont été estimées. Au total, 203 placettes permanentes ont été délimitées, 48 placettes aux abords de chemin, 50 sous les fils de hautetension, 40 à la périphérie du parc, 23 dans les campements, 22 dans l'arboretum et 20 dans la forêt d'Anguédédou. Les placettes installées sous les lignes de haute tension, aux abords de chemin et à la périphérie du parc sont équidistantes de $200 \mathrm{~m}$ et celles installées dans l'arboretum, les campements et la portion de forêt d'Anguédédou sont équidistantes de $50 \mathrm{~m}$.

\section{Critères d'identification des espèces invasives}

Dans le cadre de cette étude, une proposition de liste de plantes invasives a été élaborée sur la base de certaines listes existantes d'Espèces Exotiques Envahissantes (EEE) de Côte d'Ivoire. En effet, des ouvrages et articles publiés ou non publiés, des rapports sur les plantes invasives ont été consultés (De Foresta, 1995 ; Gautier et al., 1999 ; Chatelain et al, 2001 ; Neuba et al., 2014 ; UICN/PACO, 2013 ; Noba et al., 2017), de même que la liste établie par le Professeur Aké-Assi (données personnelles) sur les plantes invasives de Côte d'Ivoire. La base de données mondiale des espèces invasives (www.issg.org/database/welcome), le recueil d'espèces invasives de CABIwww.cabi.org/isc/ et la liste des plantes invasives de l'Afrique de l'ouest (http://issg.org/pdf/puplications/GISP/Resources/ wAfrica-EN.pdf) ont été également consultés. Ces données ont été complétées par des communications personnelles d'experts locaux et des observations personnelles. Cette liste provisoire nous a servi de support pour l'inventaire des espèces invasives du Parc National du Banco. 


\section{Classification des espèces invasives}

Les espèces invasives du Parc National du Banco ont ensuite été classifiées selon la méthode de Dortel \& Geslin (2016) et Quere \& Geslin (2016). D'après cette classification, il existe trois catégories de plantes invasives. Les invasives avérées (IA) : plantes non indigènes montrant une dynamique d'extension rapide du fait d'une reproduction sexuée ou d'une multiplication végétative intenses, et formant localement, des populations denses et bien installées; les plantes invasives potentielles (IP) qui sont des espèces non indigènes et ne présentant pas actuellement de caractère envahissant avéré dans le territoire considéré mais dont la dynamique à l'intérieur de ce territoire et/ou dans des régions limitrophes ou climatiquement proches, est telle qu'il existe un risque de les voir devenir à plus ou moins long terme des espèces invasives avérées ; les plantes à surveiller (AS) : espèce non indigène ne présentant actuellement pas (ou plus) de caractère envahissant avéré mais dont la possibilité de développer un caractère envahissant n'est pas totalement écartée, compte tenu notamment du caractère envahissant de cette plante dans d'autres régions du monde.

\section{Fréquence relative des espèces invasives}

La fréquence relative ( $\mathrm{Fr}$ ) est une valeur qui traduit la régularité de la distribution d'une espèce dans une communauté végétale (Raunkiaer, 1905). Elle est calculée selon la formule suivante :

$$
\mathrm{Fr}=\frac{\mathrm{Fa}}{-----}
$$

Où $\mathrm{Fa}$ : Fréquence absolue de l'espèce et $\mathrm{N}$ : nombre d'échantillon élémentaire.

Dans la présente étude, 5 classes de fréquence relative définies selon Raunkiaer (1905) ont été utilisées pour déterminer la régularité des espèces invasives. La classe I regroupe les espèces très peu régulières dont la fréquence relative est comprise entre 0 et $20 \%$; La classe II renferme les espèces peu régulières dont la fréquence relative est comprise entre 21 et $40 \%$; La classe III des espèces régulières, la classe IV des espèces très régulières et la classe $\mathrm{V}$ des espèces particulièrement régulières regroupent respectivement les espèces dont les fréquences sont comprises entre 41 et $60 \%$; entre 61 et $80 \%$ et entre 81 et $100 \%$. 


\section{Contribution spécifique des espèces invasives}

La contribution spécifique (Cs) est l'expression de l'apport de chacune de ces espèces à la végétation d'une station inventoriée. Ce paramètre a été calculé pour mettre en évidence l'agressivité des espèces invasives. Elle s'obtient à partir de la formule proposée par Daget \& Poissonet (1969) :

$$
\begin{gathered}
F S(\mathrm{e}) \\
100
\end{gathered}
$$

Où $F S(e)$ : fréquence absolue de l'espèce considérée. Cette étude a adopté la classification de Daget \& Poissonet (1969) :

- Cs $($ e $)<1$ : espèces dont l'effet dépressif sur les plantes est plus ou moins négligeable ;

$-1 \leq \mathrm{Cs}(\mathrm{e})<4$ : espèces dites agressives, dont l'effet dépressif sur les plantes est relativement élevé, elles sont dites agressives ;

- Cs (e) $\geq 4$ : espèces considérées comme très agressives vis-à-vis des autres plantes ; elles ont un effet dépressif particulièrement élevé sur les autres espèces.

\section{Dynamique d'expansion des espèces invasives}

L'étude de la dynamique d'expansion des espèces invasives a été réalisée à travers deux approches. La première a consisté à réaliser la carte de distribution des espèces. Pour cela, les coordonnées géographiques des différentes espèces invasives prises sur le terrain ont été superposées à la carte du PNB. Le logiciel QGIS 2.14 a été utilisé à cet effet. La seconde approche a portée sur l'analyse de la dynamique de colonisation des biotopes sur une période de trois années consécutives (2016, 2017 et 2018). La surface d'occurrence des espèces $(\mathrm{Sp})$ a été calculée suivant la formule proposée par Scherer (1984) : utilisée par Nzengue et al. (2016) et Anzil et al. (2017).

$$
S p=\sum_{i=1}^{n} X i
$$

Avec $\mathrm{X} i$ : superficie représentant l'occurrence de chaque zone d'occurrence ou surface élémentaire de la zone d'occurrence; n: le nombre de zone d'occurrence.

La surface d'occupation réelle des espèces invasives a été évaluée en multipliant à la surface d'occurrence par le taux de recouvrement. Elle a été calculée selon la formule suivante de Scherer (1984) : utilisée par Nzengue et al. (2016) et Anzil et al. (2017). 


$$
S r=\sum_{i=1}^{n} X i . C i
$$

Avec $\mathrm{Ci}$ : taux de recouvrement du peuplement d'une espèce invasive.

Par la suite, la surface totale annuelle occupée par chaque espèce a été obtenue en faisant la somme des surfaces d'occupation de chaque biotope. Le taux d'expansion des espèces invasives a été calculé afin d'estimer l'évolution (croissance ou régression) de la superficie occupée par ces espèces sur les trois années consécutives. Sa formule mathématique est :

$\mathrm{TC}=\left(\mathrm{S}_{\mathrm{f}}-\mathrm{S}_{\mathrm{i}} / \mathrm{S}_{\mathrm{i}}\right) * 100$.

TC est le taux de croissance ou d'expansion. $S_{\mathrm{i}}$ est la superficie d'occupation du sol des plantes invasives de l'année initiale et $S_{\mathrm{f}}$ représente la superficie d'occupation du sol de l'année finale.

\section{Résultats}

\section{Richesse spécifique}

Cette étude a permis de recenser au total 302 espèces végétales reparties en 220 genres et 93 familles. Dans cette flore, 22 espèces ont été identifiées comme invasives. Une espèce avérée invasive (Chromolaena odorata) et 21 espèces invasives potentielles ont été répertoriées (Tableau 1). Chromolaena odorata est présent dans trois zones de présence, sous les fils de haute tension, dans les campements et à la périphérie du parc. Les invasives potentielles ont été rencontrées dans toutes les zones de présence. Huit, quatorze et treize invasives potentielles ont été rencontrées respectivement dans les mêmes zones de présence que Chromolaena odorata. Il a aussi été recensé cinq espèces invasives potentielles en bordures des routes, trois dans l'arboretum et quatre dans la forêt d'Anguédédou. 
Tableau 1 : Liste, forme de vie, origine et statut des plantes invasives avérés et invasives potentielles du Parc National su Banco

Espèces

Chromolaena odorata

King \& H. Rox.

Ageratum conyzoides L.

Alternanthera brasiliana (L.) Kuntze

Bambusa vulgaris Schrad. ex J. C. Wendel.

Breynia disticha J. R. \& G. Forst

Cecropia peltata Linn.

Cedrela toona Rox.ex Wild

Croton hirtus L'Hérit.

Dieffenbachia picta Schott

Euphorbia heterophylla Linn.

Gmelina arborea Rox.

Hopea odorata Rox.

Lantana camara Linn.

Leucaena leucocephala (Lam.) De Wilt

Mimosa invisa Martius ex Colla.

Pueraria phaseoloides (Rox.) Benth.

Solanum erianthum D. Don

Solanum rugosum Dun.

Stenotaphrum secundatum (Walter) Kuntze

Syngonium podophylum Schott

Tithonia diversifolia A. Gray

Turnera ulmifolia L.
Familles

Asteraceae

Asteraceae

Amaranthaceae

Poaceae

Euphorbiaceae

Cecropiaceae

Meliaceae

Euphorbiaceae

Araceae

Euphorbiaceae

Verbenaceae

Dipterocarpaceae

Verbenaceae

Fabaceae

Fabaceae

Fabaceae

Solanaceae

Solanaceae

Poaceae

Araceae

Asteraceae

Passifloraceae
Forme de vie

arbuste

herbe

herbe

herbe

arbuste

arbre

arbre

herbe

herbe

herbe

arbre

arbre

arbuste

arbre

liane

liane

arbuste

arbuste

herbe

herbe

arbuste

liane
Origine

Amérique centrale

Amérique centrale

Brésil

Indochine

Iles du pacifique

Amérique tropicale

Chine

Amérique tropicale

Amérique centrale

Amérique centrale

Pakistan

Asie du Sud-Est

Antilles

Amérique tropicale

Amérique du sud

Extrême orient

Amérique centrale

Amérique du Sud

Europe

Amérique centrale

Amérique centrale

Amérique centrale
Statut

Avérée invasive

Invasive potentielle

Invasive potentielle Invasive potentielle Invasive potentielle Invasive potentielle Invasive potentielle Invasive potentielle Invasive potentielle Invasive potentielle Invasive potentielle Invasive potentielle Invasive potentielle Invasive potentielle Invasive potentielle Invasive potentielle Invasive potentielle Invasive potentielle Invasive potentielle Invasive potentielle Invasive potentielle Invasive potentielle

\section{Fréquence relative des espèces invasives}

Chromolaena odorata est l'espèce la plus fréquente sous les fils de haute tension, dans les campements et à la périphérie du parc, Elle est très régulière avec des fréquences relatives respectives de 71,05\%,64,28 \% et $78,26 \%$ (Tableau 2).

Trois espèces invasives potentielles sont fréquentes sous les fils de haute tension avec des fréquences relatives respectives de 55,26\% Ce sont Cecropia peltata, Lantana camara et Mimosa invisa. Les espèces les moins fréquentes sont Ageratum conizoides et Bambusa vulgaris qui ont chacune une fréquence relative de $7,89 \%$. 
Breynia disticha et Cecropia peltata sont les espèces les plus fréquentes dans les campements, avec une fréquence relative de 52,38\%, chacune. Syngonium podophylum et Turnera ulmifolia sont les deux espèces les moins fréquentes avec des fréquences relatives respectivement de 26,19\%.

A la périphérie du parc, Pueraria phaseoloides $(73,9 \%)$ et Breynia disticha $(69,56 \%)$ sont les espèces les plus fréquentes. L'espèce la moins fréquente est Ageratum conizoides avec une fréquence relative de 8,69\%.

En bordures des routes, toutes les espèces invasives potentielles sont moins fréquentes. Ce sont, par ordre d'importance Ageratum conizoides (10,27\%), Hopea odorata (9\%) Bambusa vulgaris (2,27\%), Cecropia peltata $(2,27 \%)$ et Alternanthera brasiliana (2,27 \%). Dans l'arboretum et la forêt d'Anguédédou, Hopea odorata est très fréquente avec une fréquence relative respective de $72,72 \%$ et $77,77 \%$.

\section{Contribution spécifique des espèces invasives}

La seule espèce avérée invasive, Chromolaena odorata, est très agressive dans les campements, sous les fils de haute tension et à la périphérie du parc avec une contribution spécifique de 12,08; 10,17; et 7,09 respectivement (Tableau 3). Aux abords des routes, toutes les espèces invasives potentielles ont une contribution spécifique comprise entre 1 et 4 . Ageratum conizoides est l'espèce qui a la plus forte valeur de contribution spécifique (3,02), suivie de Bambusa vulgaris, Cecropia peltata et Hopea odorata qui ont comme valeurs de contribution spécifique respectivement 1,$62 ; 1,48$ et 1,51. Dans l'arboretum et dans la forêt d'Anguédédou, Hopea odorata est la seule espèce très agressive avec une contribution spécifique supérieure à 4 . Les autres espèces ont une contribution spécifique qui varie entre 1 et 4 . 
Tableau 2 : Fréquences relatives des espèces invasives recensées dans le Parc National su Banco

\begin{tabular}{|c|c|c|c|c|c|c|c|c|c|c|c|}
\hline \multicolumn{2}{|c|}{ Bord de route } & \multicolumn{2}{|c|}{ Haute-tension } & \multicolumn{2}{|c|}{ Campement } & \multicolumn{2}{|c|}{ Arboretum } & \multicolumn{2}{|c|}{ Périphérie } & \multicolumn{2}{|c|}{ Forêt } \\
\hline Espèces & Fr & Espèces & Fr & Espèces & Fr & Espèces & Fr & Espèces & Fr & Espèces & $\mathrm{Fr}$ \\
\hline $\begin{array}{l}\text { Ageratum } \\
\text { conizoides }\end{array}$ & 10,27 & $\begin{array}{l}\text { Chromolaena } \\
\text { odorata }\end{array}$ & 71,05 & $\begin{array}{l}\text { Chromolaena } \\
\text { odorata }\end{array}$ & 64,28 & $\begin{array}{l}\text { Hopea } \\
\text { odorata }\end{array}$ & 72,72 & $\begin{array}{l}\text { Chromolaena } \\
\text { odorata }\end{array}$ & 78,26 & $\begin{array}{l}\text { Hopea } \\
\text { odorata }\end{array}$ & 77,77 \\
\hline $\begin{array}{l}\text { Hopea } \\
\text { odorata }\end{array}$ & 9 & $\begin{array}{l}\text { Cecropia } \\
\text { peltata }\end{array}$ & 55,26 & $\begin{array}{l}\text { Breynia } \\
\text { disticha }\end{array}$ & 52,28 & $\begin{array}{l}\text { Breynia } \\
\text { disticha }\end{array}$ & 45,45 & $\begin{array}{l}\text { Pueraria } \\
\text { phaseloides }\end{array}$ & 73,91 & $\begin{array}{l}\text { Bambusa } \\
\text { vulgaris }\end{array}$ & 68,68 \\
\hline $\begin{array}{l}\text { Bambusa } \\
\text { vulgaris }\end{array}$ & 2,27 & $\begin{array}{l}\text { Lantana } \\
\text { camara }\end{array}$ & 55,26 & $\begin{array}{l}\text { Cecropia } \\
\text { peltata }\end{array}$ & 52,28 & $\begin{array}{l}\text { Bambusa } \\
\text { vulgaris }\end{array}$ & 18,18 & $\begin{array}{l}\text { Breynia } \\
\text { disticha }\end{array}$ & 69,56 & $\begin{array}{l}\text { Gmelina } \\
\text { arborea }\end{array}$ & 53,33 \\
\hline $\begin{array}{l}\text { Cecropia } \\
\text { peltata }\end{array}$ & 2,27 & $\begin{array}{l}\text { Mimosa } \\
\text { invisa }\end{array}$ & 55,26 & $\begin{array}{l}\text { Mimosa } \\
\text { invisa }\end{array}$ & 40,46 & & & $\begin{array}{l}\text { Stenotaphrum } \\
\text { secundatum }\end{array}$ & 47,82 & $\begin{array}{l}\text { Cedrela } \\
\text { odorata }\end{array}$ & 48,88 \\
\hline $\begin{array}{l}\text { Alternanthera } \\
\text { brasiliana }\end{array}$ & 2,27 & Croton hirtus & 18,42 & $\begin{array}{l}\text { Stenotaphrum } \\
\text { secundatum }\end{array}$ & 38,09 & & & $\begin{array}{l}\text { Mimosa } \\
\text { invisa }\end{array}$ & 60,86 & & \\
\hline & & $\begin{array}{l}\text { Breynia } \\
\text { disticha }\end{array}$ & 15,78 & $\begin{array}{l}\text { Pueraria } \\
\text { phaseloides }\end{array}$ & 38,09 & & & $\begin{array}{l}\text { Lantana } \\
\text { camara }\end{array}$ & 56,52 & & \\
\hline & & $\begin{array}{l}\text { Bambusa } \\
\text { vulgaris }\end{array}$ & 7,79 & $\begin{array}{l}\text { Hopea } \\
\text { odorata }\end{array}$ & 35,71 & & & Croton hirtus & 52,17 & & \\
\hline & & $\begin{array}{l}\text { Ageratum } \\
\text { conizoides }\end{array}$ & 7,29 & $\begin{array}{l}\text { Lantana } \\
\text { camara }\end{array}$ & 35,71 & & & $\begin{array}{l}\text { Hopea } \\
\text { odorata }\end{array}$ & 43,87 & & \\
\hline & & $\begin{array}{l}\text { Titholia } \\
\text { diversifolia }\end{array}$ & 7,29 & $\begin{array}{l}\text { Dieffenbachia } \\
\text { picta }\end{array}$ & 33,33 & & & $\begin{array}{l}\text { Titholia } \\
\text { diversifolia }\end{array}$ & 43,47 & & \\
\hline & & & & $\begin{array}{l}\text { Solanum } \\
\text { rugosum }\end{array}$ & 33,33 & & & $\begin{array}{l}\text { Solanum } \\
\text { rugosum }\end{array}$ & 39,13 & & \\
\hline & & & & $\begin{array}{l}\text { Alternanthera } \\
\text { brasiliana }\end{array}$ & 33,33 & & & $\begin{array}{l}\text { Solanum } \\
\text { erianthum }\end{array}$ & 30,43 & & \\
\hline & & & & $\begin{array}{l}\text { Bambusa } \\
\text { vulgaris }\end{array}$ & 30,95 & & & $\begin{array}{l}\text { Bambusa } \\
\text { vulgaris }\end{array}$ & 30,43 & & \\
\hline & & & & Croton hirtus & 30,95 & & & $\begin{array}{l}\text { Leucena } \\
\text { leucocephala }\end{array}$ & 30,43 & & \\
\hline & & & & $\begin{array}{l}\text { Euphorbia } \\
\text { heterophylum }\end{array}$ & 26,19 & & & $\begin{array}{l}\text { Turnera } \\
\text { ulmifolia }\end{array}$ & 8,69 & & \\
\hline & & & & $\begin{array}{l}\text { Syngonium } \\
\text { podophylum }\end{array}$ & 26,19 & & & & & & \\
\hline
\end{tabular}


Tableau 3 : Contribution spécifique des espèces invasives recensées dans le Parc National su Banco

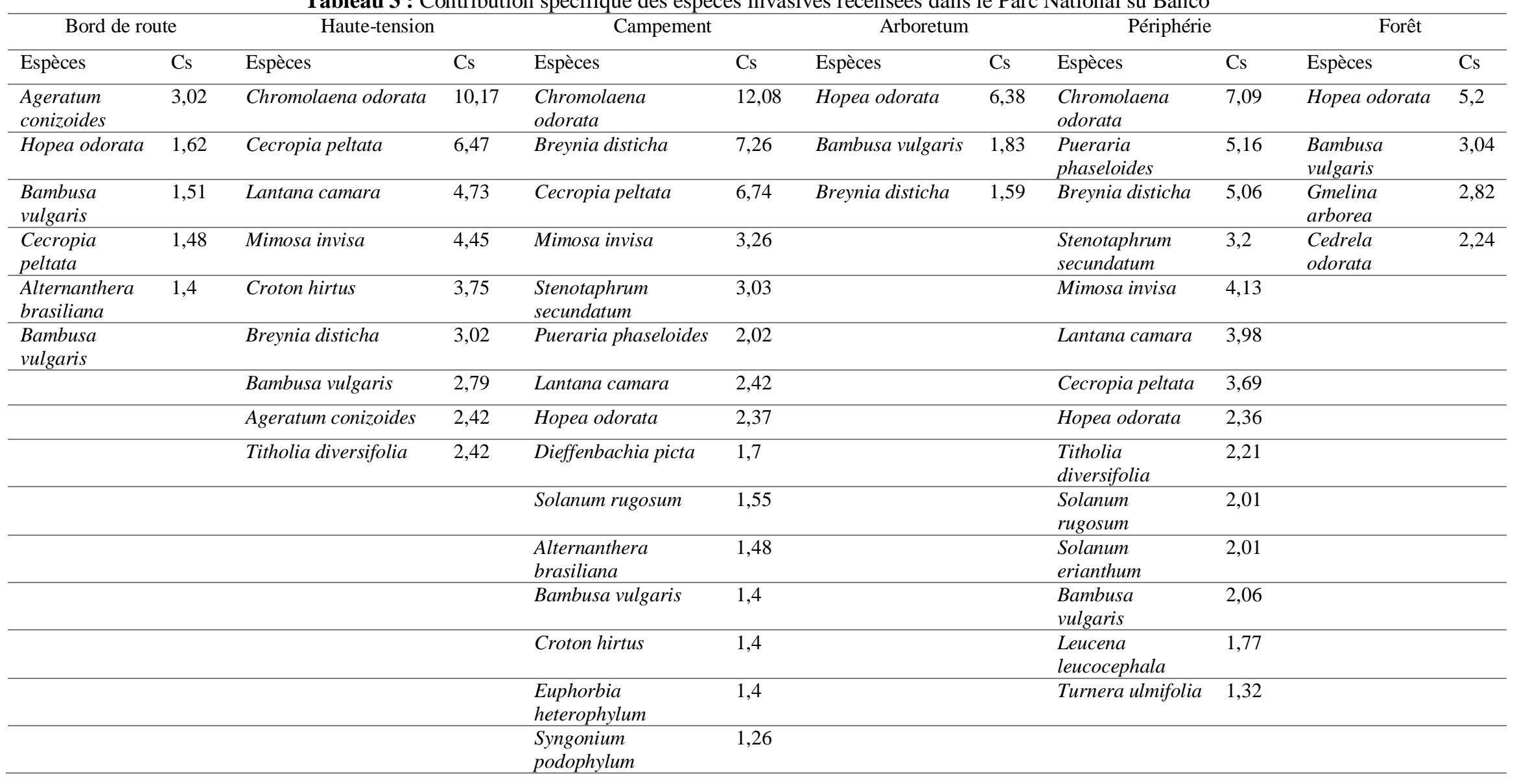




\section{Dynamique spatio-temporelle des espèces invasives du PNB}

La carte de distribution des plantes invasives montre que ces espèces se répartissent dans l'ensemble du parc (Figure 1). En 2016, elles occupent dans les zones rudérales une surface au sol de 6,1 ha, ce qui correspond à 0,17 $\%$ de la surface totale du parc (Tableau 4). On note une concentration plus marquée dans les campements et à la périphérie avec un taux de couverture de plus de $65 \%$. Chromolaena odorata et Bambusa vulgaris sont les espèces les plus recouvrantes avec une surface au sol respective de 0,20 et 0,23 ha dans les campements. Elles couvrent aussi respectivement 1,21 et 0,9 ha de la périphérie du parc. Sous les fils de haute tension, les espèces invasives ont un taux de couverture de $45,5 \%$.

Chromolaena odorata et Bambusa vulgaris sont également les plus recouvrantes avec une surface au sol respective de 1,5 et 0,6 ha. En bordure des routes et dans l'arboretum, les plantes invasives sont peu recouvrantes avec un taux de couverture respectif de 36,5 et 20,8 \% . Bambusa vulgaris occupe 0,31 ha des bords de routes et Hopea odorata occupe une surface au sol de 0,30 ha dans l'arboretum. Dans la forêt d'Anguédédou, la surface au sol de la plantation de Hopea odorata est de 76,71 ha.

En 2017, les zones rudérales du parc sont colonisées par les plantes invasives avec une surface du sol de 7,14 ha soit $0,20 \%$ de la surface totale du parc (Tableau 4). Ce qui correspond à un taux d'accroissement de 69,04\% (Tableau 5). Dans les campements et à la périphérie, le taux de couverture des espèces invasives est plus de $75 \%$ (Tableau 4). Chromolaena odorata et Bambusa vulgaris sont toujours les espèces qui colonisent le plus d'espace. Sous les fils de haute tension, les espèces invasives ont un taux de couverture de $49 \%$. Chromolaena odorata et Bambusa vulgaris sont également les plus recouvrantes avec une surface au sol respective de 0,51 et 1,6 ha. Dans l'arboretum et en bordure de route, les espèces invasives occupent une surface au sol de 0,5 et 0,36 ha respectivement. Chromolaena odorata et Bambusa vulgaris sont également les espèces qui colonisent le plus d'espace Dans la forêt d'Anguédédou, la surface au sol de la plantation de Hopea odorata est de 82,62 ha. 
Tableau 4 : Surfaces occupées par les plantes invasives dans le Parc National su Banco en 2016, 2017 et 2018

\begin{tabular}{|c|c|c|c|c|c|c|c|c|c|c|}
\hline \multicolumn{2}{|c|}{ Zone de présence } & \multicolumn{3}{|l|}{2016} & \multicolumn{3}{|l|}{2017} & \multicolumn{3}{|l|}{2018} \\
\hline \multirow{7}{*}{$\begin{array}{l}\text { Bord de } \\
\text { route }\end{array}$} & Espèces & $\begin{array}{l}\text { Surf. prés } \\
\text { (ha) }\end{array}$ & $\begin{array}{l}\text { Taux de } \\
\text { couv }(\%)\end{array}$ & $\begin{array}{l}\text { Surf. } \\
\text { d'occ (ha) }\end{array}$ & $\begin{array}{l}\text { Surf. } \\
\text { prés (ha) }\end{array}$ & $\begin{array}{l}\text { Taux de } \\
\operatorname{couv}(\%)\end{array}$ & $\begin{array}{l}\text { Surf. } \\
\text { d'occ (ha) }\end{array}$ & $\begin{array}{l}\text { Surf. } \\
\text { prés (ha) }\end{array}$ & $\begin{array}{l}\text { Taux de } \\
\text { couv }(\%)\end{array}$ & $\begin{array}{l}\text { Surf. } \\
\text { d'occ (ha) }\end{array}$ \\
\hline & $\begin{array}{l}\text { Ageratum } \\
\text { conizoides }\end{array}$ & 0,02 & 3,5 & 0 & 0,05 & 3,5 & 0 & 0,55 & 3,5 & 0,02 \\
\hline & Bambusa vulgaris & 2,5 & 12,5 & 0,31 & 2,5 & 12,5 & 0,31 & 2,7 & 20 & 0,7 \\
\hline & Hopea odorata & 0,12 & 2,5 & 0 & 0,2 & 3 & 0,33 & 0,2 & 3 & 0 \\
\hline & Cecropia peltata & 0,25 & 1,3 & 0 & 0,5 & 1,6 & 0 & 0,25 & 12,5 & 0,31 \\
\hline & $\begin{array}{l}\text { Alternanthera } \\
\text { brasiliana }\end{array}$ & 0,02 & 1 & 0 & 0,03 & 1 & 0,03 & 0 & 0 & 0 \\
\hline & Total & 2,9 & 20,8 & $\mathbf{0 , 3 1}$ & 3,28 & 21,6 & 0,36 & 3,2 & 39 & 1,03 \\
\hline \multirow{9}{*}{$\begin{array}{l}\text { Haute- } \\
\text { tension }\end{array}$} & $\begin{array}{l}\text { Chromolaena } \\
\text { odorata }\end{array}$ & 6,53 & 9 & 0,6 & 6,07 & 8,5 & 0,51 & 5 & 8 & 0,4 \\
\hline & Croton hirtus & 0,6 & 1,5 & 0,01 & 0,6 & 1,5 & 0,01 & 0,8 & 2 & 0,02 \\
\hline & Breynia distica & 1,2 & 2 & 0,02 & 1,5 & 2 & 0,03 & 1,96 & 3,5 & 0,7 \\
\hline & Bambusa vulgaris & 6,2 & 24 & 1,5 & 6,4 & 25 & 1,6 & 6,4 & 25 & 1,6 \\
\hline & Cecropia peltata & 0,61 & 1,5 & 0,01 & 0,7 & 2 & 0,01 & 2,44 & 5 & 0,12 \\
\hline & Lantana camara & 1,16 & 4 & 0,04 & 1,16 & 4 & 0,05 & 1,2 & 3,5 & 0,4 \\
\hline & Mimosa invisa & 2,1 & 1 & 0,02 & 2,2 & 1 & 0,02 & 2,2 & 1 & 0,02 \\
\hline & $\begin{array}{l}\text { Titholia } \\
\text { diversifolia }\end{array}$ & 0,31 & 1 & 0,01 & 0,9 & 3 & 0,03 & 1,3 & 4 & 0,05 \\
\hline & Total & 19,01 & 45,5 & 2,4 & 20,05 & 49 & 2,81 & 22,83 & 56 & 3,91 \\
\hline \multirow{3}{*}{ Campement } & $\begin{array}{l}\text { Chromolaena } \\
\text { odorata }\end{array}$ & 1,03 & 20,2 & 0,2 & 1,08 & 21,2 & 0,22 & 3,05 & 22,2 & 0,8 \\
\hline & Breynia distica & 0,02 & 2,02 & 0 & 0,02 & 2,02 & 0 & 0,02 & 3,02 & 0 \\
\hline & Cecropia peltata & 0,05 & 1,46 & 0 & 0,05 & 1,46 & 0 & 0,05 & 1,46 & 0 \\
\hline
\end{tabular}




\begin{tabular}{|c|c|c|c|c|c|c|c|c|c|c|}
\hline \multirow{13}{*}{ Campement } & Mimosa invisa & 0,23 & 1,61 & 0 & 0,23 & 1,61 & 0 & 0,23 & 1,61 & 0 \\
\hline & $\begin{array}{l}\text { Stenotaphrum } \\
\text { secundatum }\end{array}$ & 0,8 & 10,12 & 0,1 & 0,8 & 13,12 & 0,16 & 0,8 & 20,5 & 0,03 \\
\hline & $\begin{array}{l}\text { Pueraria } \\
\text { phaseoloides }\end{array}$ & 0,02 & 1,66 & 0 & 0,02 & 1,66 & 0 & 0,02 & 1,66 & 0 \\
\hline & Lantana camara & 0,23 & 3,5 & 0 & 0,23 & 3,5 & 0 & 0,23 & 3,5 & 0 \\
\hline & Hopea odorata & 0,23 & 1,5 & 0 & 1,6 & 1,5 & 0 & 0,99 & 1,5 & 0,1 \\
\hline & $\begin{array}{l}\text { Dieffenbachia } \\
\text { picta }\end{array}$ & 0,02 & 1 & 0 & 0,02 & 1 & 0 & 0,02 & 1 & 0 \\
\hline & Solanum rugosum & 0,23 & 1,83 & 0 & 0,23 & 1,83 & 0 & 0,23 & 1,83 & 0 \\
\hline & $\begin{array}{l}\text { Alternanthera } \\
\text { brasiliana }\end{array}$ & 0,01 & 2,25 & 0 & 0,01 & 2,25 & 0 & 0,01 & 2,25 & 0 \\
\hline & Bambusa vulgaris & 1,23 & 17,25 & 0,23 & 1,23 & 20,25 & 0,3 & 4,23 & 28 & 1,26 \\
\hline & Croton hirtus & 0,01 & 1,5 & 0 & 0,01 & 1,5 & 0 & 0,01 & 1,5 & 0 \\
\hline & $\begin{array}{l}\text { Euphorbia } \\
\text { heterophylla }\end{array}$ & 0,02 & 2 & 0 & 0,02 & 2 & 0 & 0,02 & 2 & 0 \\
\hline & $\begin{array}{l}\text { Syngonium } \\
\text { podophylum }\end{array}$ & 0,06 & 1,5 & 0 & 0,06 & 1,8 & 0 & 0,06 & 1,8 & 0 \\
\hline & Total & 4,19 & 69,9 & $\mathbf{0 , 5 3}$ & 5,61 & 77,2 & $\mathbf{0 , 7}$ & 6,1 & 93,83 & 2,46 \\
\hline \multirow{4}{*}{ Arboretum } & Hopea odorata & 1,5 & 20,5 & 0,3 & 1,6 & 21,5 & 0,34 & 1,6 & 21,5 & 0,35 \\
\hline & Bambusa vulgaris & 1,24 & 12,5 & 0,16 & 1,24 & 12,5 & 0,16 & 1,76 & 12,5 & 0,22 \\
\hline & Breynia distica & 0,06 & 3,5 & 0 & 0,12 & 3,5 & 0 & 0,28 & 9,5 & 0,03 \\
\hline & Total & 2,8 & 36,5 & 0,46 & 2,96 & 37,5 & 0,5 & 3,66 & 43,5 & 0,6 \\
\hline \multirow{4}{*}{ Périphérie } & $\begin{array}{l}\text { Chromolaena } \\
\text { odorata }\end{array}$ & 4,03 & 30,2 & 1,21 & 4,5 & 31,2 & 1,4 & 6,44 & 35,2 & 2,26 \\
\hline & $\begin{array}{l}\text { Pueraria } \\
\text { phaseoloides }\end{array}$ & 1,18 & 2,02 & 0,02 & 2,5 & 2,02 & 0,05 & 2,5 & 2,02 & 0,05 \\
\hline & Breynia distica & 1,1 & 1,46 & 0,02 & 1,05 & 1,46 & 0,02 & 1,44 & 1,46 & 0,02 \\
\hline & Mimosa invisa & 0,16 & 1,61 & 0 & 0,26 & 1,61 & 0 & 1,04 & 1,61 & 0,01 \\
\hline
\end{tabular}




\begin{tabular}{|c|c|c|c|c|c|c|c|c|c|c|}
\hline & Lantana camara & 1,16 & 13,12 & 0,15 & 1,16 & 13,12 & 0,16 & 1,44 & 16,12 & 0,2 \\
\hline & $\begin{array}{l}\text { Stenotaphrum } \\
\text { secundatum } \\
\end{array}$ & 1,16 & 1,66 & 0,02 & 1,16 & 1,66 & 0,02 & 1,44 & 1,66 & 0,02 \\
\hline & Cecropia peltata & 1,16 & 3,5 & 0,04 & 1,06 & & & 2,34 & 3,5 & 0,09 \\
\hline & Hopea odorata & 1,16 & 1,5 & 0,02 & 1,16 & 1,5 & 0,02 & 1,5 & 1,5 & 0,03 \\
\hline & $\begin{array}{l}\text { Titholia } \\
\text { diversifolia }\end{array}$ & 0,16 & 0,5 & 0 & 1,16 & 0,5 & 0 & 1,44 & 0,5 & 0 \\
\hline & Solanum rugosum & 0,25 & 1,83 & 0 & 0 & 0 & 0 & 0 & 0 & 0 \\
\hline & $\begin{array}{l}\text { Solanum } \\
\text { erianthum }\end{array}$ & 0,02 & 2,25 & 0 & 0 & 2,25 & 0 & 0 & 0 & 0 \\
\hline & Bambusa vulgaris & 5,16 & 18,25 & 0,9 & 5,16 & 20,25 & 1,04 & 6,44 & 20 & 1,3 \\
\hline & $\begin{array}{l}\text { Leucena } \\
\text { leucocephala }\end{array}$ & 1,16 & 1,5 & 0,02 & 1,16 & 2,5 & 0,03 & 1,06 & 1,5 & 0,03 \\
\hline & $\begin{array}{l}\text { Euphorbia } \\
\text { heterophylla }\end{array}$ & 0,32 & 2 & 0 & 0 & 0 & 0 & 0 & 0 & 0 \\
\hline & Total & 18,18 & 81,4 & 2,4 & 20,23 & 84,03 & 2,77 & 27,08 & 85,07 & 4,01 \\
\hline & $\begin{array}{l}\text { Surface totale } Z \text {. } \\
\text { rudérale }\end{array}$ & 47,08 & 50,82 & 6,1 & 52,13 & 53,86 & 7,14 & 62,87 & 63,48 & 12,01 \\
\hline \multirow{5}{*}{ Forêts } & Hopea odorata & 180 & 42,62 & 76,71 & 183,50 & 47,75 & 87,62 & 185 & 57,75 & 106,83 \\
\hline & Gmelina arborea & 0,14 & 1,5 & 0 & 0,14 & 1,5 & 0 & 0,14 & 1,5 & 0 \\
\hline & Cedrela toona & 0,02 & 1 & 0 & 0,02 & 1 & 0 & 0,02 & 1 & 0 \\
\hline & total & 180,16 & 45,12 & 76,71 & 183,66 & 49,80 & 87,62 & 185,16 & 60,25 & 106,83 \\
\hline & Surface total & 227,24 & & 82,81 & 235,79 & & 94,76 & 248,03 & & 126,84 \\
\hline
\end{tabular}


En 2018, 12,01 ha (0,34\%) est occupé par les plantes invasives dans les zones rudérales (Tableau 4). Ce qui correspond à un taux d'accroissement de 96,68 \% (Tableau 5). Dans les campements et à la périphérie, elles ont un taux de couverture de plus $80 \%$ (Tableau 4). Chromolaena odorata et Bambusa vulgaris demeurent les espèces les plus recouvrantes avec respectivement 0,8 et 1,26 ha d'occupation du sol dans les campements. Elles occupent aussi respectivement 2,26 et 1,3 ha de la périphérie du parc. Sous les fils de haute tension, les plantes invasives couvrent 3,91 ha. Dans l'arboretum, elles ont une surface d'occupation du sol de 0,6 ha. Hopea odorata et Bambusa vulgaris y sont les plus recouvrantes avec une surface de 0,35 et 0,22 ha respectivement. En bordure de routes, les plantes invasives couvrent 1,03 ha. Bambusa vulgaris et Cecropia peltata occupent respectivement une surface au sol de 0,7 et 0,31 ha. Dans la forêt d'Anguédédou, la surface au sol de la plantation de Hopea odorata est de 106,83 ha.

Tableau 5 : surfaces occupées et taux d'expansion des plantes invasives dans le Parc National du Banco de 2016 à 2018

\begin{tabular}{|c|c|c|c|c|c|c|}
\hline \multirow[t]{2}{*}{ Zone de présence } & \multicolumn{3}{|c|}{ Surface d'occupation (ha) } & \multicolumn{3}{|c|}{ Taux d'expansion } \\
\hline & 2016 & 2017 & 2018 & $\begin{array}{l}2016- \\
2017\end{array}$ & $\begin{array}{l}2017- \\
2018\end{array}$ & $\begin{array}{c}2016- \\
2018\end{array}$ \\
\hline Haute tension & 2,4 & 2,8 & 3,9 & 16,66 & 39,28 & 62,5 \\
\hline Arboretum & 0,46 & 0,5 & 0,6 & 8,69 & 20 & 30,43 \\
\hline Campement & 0,53 & 0,7 & 2,5 & 32,07 & 257,14 & 371,69 \\
\hline Bord de route & 0,31 & 0,4 & 1,03 & 29,03 & 157,5 & 232,25 \\
\hline Périphérie & 2,4 & 2,8 & 4,01 & 16,66 & 43,21 & 67,03 \\
\hline Total surf. Z. rudérale & 6,1 & 7,14 & 12,01 & 17,04 & 69,04 & 96,68 \\
\hline Forêt plantée & $\begin{array}{l}76,7 \\
1\end{array}$ & 87,62 & 106,83 & 14,22 & 21,92 & 39,26 \\
\hline Surface totale & $\begin{array}{l}82,8 \\
1\end{array}$ & 94,76 & 126,84 & 14,43 & 33,85 & 53,16 \\
\hline$\%$ / surface totale du parc & 2,38 & 2,72 & 3,68 & & & \\
\hline
\end{tabular}

\section{Discussion}

L'inventaire des Espèces Exotiques Envahissantes (EEE) permet d'appuyer et d'orienter les politiques relatives à la conservation de la biodiversité et de définir les espèces nécessitant des mesures de gestion, de réglementation et de prévention visant à freiner leur extension (Dortel \& Geslin, 2016). En Côte d'Ivoire, l'évaluation du statut d'invasibilité des espèces exotiques n'est pas encore exhaustive. Des études complémentaires prenant en compte les critères définis par Roux (2011), Dortel \& Geslin (2016), Quere \& Geslin (2016) sont indispensables en vue de définir clairement une espèce invasive. Dans le cadre de cette étude, certains 
paramètres décrits par les auteurs suscités ont été analysés. A cet effet, dans le Parc National du Banco, 21 espèces sont considérées comme invasives potentielles et une seule espèce est avérée invasive, Chromolaena odorata. Cette espèce, introduite volontairement en Côte d'Ivoire en 1960, s'est rapidement répandue et est devenue une composante à part entière des végétations anthropisées (De Foresta, 1991). Aujourd'hui, selon les travaux de Neuba et al. (2014), C. odorata est présente sur toute l'étendue du territoire ivoirien, aussi bien en forêt qu'en savane. Les travaux de Maroun (2017) portant sur la perception des populations des milieux agricoles sur C. odorata, ont montré qu'elle est la mauvaise herbe la plus citée par $70 \%$ des villageois et qu'environ 60 à 70\% lui confèrent un impact négatif sur les cultures, principalement à cause de son caractère envahissant. Kouassi et al. (2008) ont également montré que des forts taux de recouvrement de $C$. odorata empêchaient l'enrichissement de la flore des jachères. Aussi, les travaux de Tiébré \& Gouli Gnanazan (2018) ont démontré que C. odorata réduit la richesse, la diversité et l'abondance des espèces indigènes dans les zones envahies du Parc National du Banco. Par ailleurs, son statut d'invasibilité a été démontré dans plusieurs pays d'Afrique notamment en Afrique de l'Ouest (Nigéria, Bénin, Ghana, Guinée, Togo, Libéria...), en Afrique centrale (Centrafrique, Cameroun, République Démocratique du Congo), en Afrique du Sud et dans plusieurs autres pays du monde comme le Laos, le Cambodge, le Vietnam, l'Indochine, l'Australie... (Mouloungou \& Sigrist, 1993 ; Waterhouse, 1994 ; Usha et al., 1996 ; Shackleton et al., 2016 ; Noba et al., 2017). Selon Lowe et al. (2007), elle fait partie des 100 espèces exotiques envahissantes les plus répandues dans le monde et les plus problématiques.

Chromolaena odorata et Hopea odorata qui une espèce potentiellement invasive, sont des espèces très régulières du PNB. Ces résultats sont en accord avec ceux de Tiébré et al. (2014). D'après ces auteurs, Hopea odorata est une espèce très régulière du PNB avec une fréquence relative de 76,92 \%. Ces deux espèces invasives sont aussi très agressives visà-vis des autres plantes du PNB. Dans le jardin botanique de l'Université Félix Houphouët-Boigny, Kouakou (2009) a aussi trouvé que Hopea odorata est très agressive avec une contribution spécifique supérieure à 4. Quant à l'agressivité de $C$. odorata vis-à-vis des autres plantes, elle a été démontrée par plusieurs auteurs sans l'utilisation de la contribution spécifique. Selon Valeri et al. (2009), l'agressivité d'une espèce invasive est l'avantage compétitif de l'espèce sur les autres. Ainsi, De Rouw (1991), Goodall et Erasmus (1996), Awanyo et al. (2011) ont montré que C. odorata produit des masses de graines à germination rapide et pousse agressivement formant une couverture épaisse sur les plantes voisines, ce qui est un autre mécanisme de l'espèce pour réprimer les plantes natives. Tiébré \& Gouli Gnanazan (2018) ont démontré que l'avantage compétitif de $C$. odorata vis-à-vis des plantes 
indigènes était lié à l'action de deux minéraux dans le sol : le potassium et le sodium échangeables. Selon ces auteurs, l'utilisation du potassium échangeable par $C$. odorata pour sa croissance entraine une carence de ce minéral pour les plantes voisines, les rendant plus sensibles à des formes de stress (gel, excès de luminosité, attaques de parasites). L’apport de sodium échangeable par l'invasion de $C$. odorata entraine une élévation de la salinité dans les milieux envahis, modifiant ainsi les propriétés physiques et mécaniques du sol au détriment des plantes voisines.

Par ailleurs, la répartition actuelle des plantes invasives sur l'ensemble du parc, justifie l'hypothèse des menaces d'invasion de ces espèces dans ce patrimoine forestier. Plusieurs études ont montré que l'invasion d'une seule espèce exotique envahissante dans les aires de conservation est très néfaste pour la biodiversité (Foxcroft et al., 2003 ; Ferraris et al., 2006 ; UICN/PACO,2013; Tiébré \& Gouli Gnanazan, 2018 ; Akodewou et al., 2019). Ces résultats corroborent les travaux de Saad et al. (2009), qui ont démontré que l'action combinée de trois espèces invasives a réduit considérablement la richesse floristique des espèces indigènes dans les réserves naturelles de Belgique et même celles des espèces à statut particulier.

L'évolution de la surface d'occupation du sol par les plantes invasives de 2016 à 2018 dans le PNB est en constante croissance. Cette évolution spatio-temporelle des espèces invasives pourrait être due aux différentes pressions anthropiques exercées par les populations riveraines chaque jour sur le parc (Hauhout, 2002 ; Sako et al., 2013 ; Sako \& Beltrando, 2014 ; Tiébré et al., 2014 ; 2015). En effet, l'accroissement des superficies envahies sous les lignes de haute tension, serait dû aux opérations de coupes fréquentes dans ces zones au cours de l'année. Cette action entraîne la dissémination des graines et des souches sur toute la surface. D'après les travaux de Walden et al. (2004), Malé et al. (2015) et Nzengue et al. (2016), certaines plantes invasives repoussent vigoureusement à partir des souches coupées et elles occupent beaucoup plus d'espace. Selon Schatz (2001), si la coupe est effectuée entre 0 à $15 \mathrm{~cm}$ au-dessus du sol, ces plantes ne meurent pas. Elles repoussent et prennent des proportions inquiétantes. A la périphérie du parc, la progression des surfaces occupées par les plantes invasives, serait liée à la pression démographique et aux activités humaines. En effet, avec le développement de la ville d'Abidjan, il y a eu une migration des populations vers la capitale économique (Hauhout, 2002). Ce mouvement a contribué à une explosion démographique et ainsi à la création des quartiers précaires autour du parc (Akoue et al., 2017). Ces populations exercent de nombreuses pressions sur le parc, notamment l'agriculture, le rejet des ordures domestiques, la pollution des eaux, etc. (Sako et Beltrando, 2014), créant ainsi les conditions favorables à la prolifération des espèces invasives. Selon Jourdan (1999), Williams et al. (2015) et Udo et al. (2016), les activités humaines représentent des foyers 
majeurs d'introduction de plantes exotiques à partir desquels certaines espèces peuvent s'étendre dans les espaces naturels environnants. En bordure des routes dans le parc et dans l'arboretum, les espèces invasives ont occupé peu d'espace. Cela peut s'expliquer par le fait que nous sommes au cœur du PNB où il y'a assez d'ombrage. Ces espèces, en général héliophile, ont suffisamment besoin de lumière pour leur germination (Salonen, 1994, Baret \& Strasberg, 2005). Par ailleurs, le développement de l'écotourisme avec les aménagements dans l'arboretum et le reprofilage des routes, pourraient constituer au cours de ces dernières années, les principales raisons de la présence des espèces invasives dans ces zones. Baret et Strasberg, (2005) ont démontré que l'élargissement de sentiers forestiers favorisent l'invasion des plantes exotiques. Ces observations sont également corroborées par les travaux de Usher (1988) et Mac Donald et al. (1989) qui ont montré que dans les aires protégées, il existe une forte corrélation entre le nombre de visiteurs et le nombre d'espèces invasives. Dans la portion de forêt d'Anguédedou, rattachée au PNB, l'espèce Hopea odorata détient une vaste surface d'occupation du sol. C'est un arbre non-indigène qui a été introduit en Côte d'Ivoire notamment dans le Parc National du Banco dans les années 1970 pour des programmes de reboisement. Les populations formées par cette espèce constituent donc les plus vieilles plantations forestières du PNB dont le sousbois et les strates moyennes s'y sont partiellement reconstitués (Lachenaud, 2006 ; Tiébré et al., 2014). Cette espèce a une grande capacité à produire des graines, se régénère énormément dans le sous-bois et à la capacité à s'étendre d'une zone à une autre (Kouakou, 2009 ; Tiébré et al., 2014). Elle est très envahissante dans la forêt d'Anguédedou où Tiébré et al. (2014) ont indiqué qu'elle avait la plus forte valeur de recouvrement moyen. Ces résultats constituent des données préliminaires sur l'inventaire et la dynamique spatiotemporelle des espèces invasives dans le Parc National du

Banco, qui pourraient être utilisées dans une politique de lutte contre ces EEE.

\section{Conclusion}

L'inventaire des plantes invasives est une nécessité dans le contexte actuel des changements globaux. Dans notre étude réalisée dans le Parc National du Banco, la diversité en plantes invasives, leur distribution de même que leur évolution ont été abordées. 22 espèces dont une espèce avérée invasive et 21 invasives potentielles ont été recensés dans la Parc National du Banco. Leur distribution se fait de manière inégale dans la PNB. Les campements, la périphérie du parc et les sites sous les lignes de haute tension sont les endroits les plus envahis, contrairement aux abords de routes et 1'arboretum. Actuellement, les Espèces Exotiques Envahissantes occupent 12,01 ha des zones rudérales du parc et, en tenant compte de la superficie plantée de Hopea odorata, 126,84 ha soit 3,65\% de la surface totale du parc. 
Les espèces comme Chromolaena odorata et Hopea odorata représentent un réel défi pour l'aire protégée. Cette étude, constitue une base pouvant servie à l'élaboration de stratégies de gestion des plantes invasives afin d'éviter l'envahissement à grande échelle du Parc National du Banco par les espèces invasives

\section{References:}

1. Abdou, A., A., M'Déré, A., Notter, J-C., \& Baret, S. (2017). Cartographie et Impacts de la liane Hiptage Benghalensis (L.) Kurz (Malpighiaceae) envahissante à la Réunion et identification des priorités de lutte, Revue d'Ecologie (Terre et Vie), 72 (2) : 147-158.

2. Akoue, Y., C., Adaman, S., \& Zon, D., A. (2017). Parc National Du Banco, Un Patrimoine Entre Destruction Et Conservation : Réalité et enjeux d'une gestion durable, European Scientific journal, 13 (2) : 1857- 7881.

3. APG III, (2009). An update of the Angiosperm Phylogeny Group classification for the orders and families of flowering plants, Botanical Journal of the Linnean Society 161: 105-121.

4. Bitty, E., A, Kadjo, B., Gonedele Bi, S., Okon, O., M., \& Kouassi, K., P. (2013). Inventaire de la faune mammalogique d'une forêt urbaine, dans le Parc National du Banco, Côte d'Ivoire, International journal of Biological and Chemical Science 7(4) : 1678- 1687.

5. Baret, S., \& Strasberg, D. (2005). The effects of opening trails on exotic plant invasion in Protected Areas on La Reunion Island (Mascarene Archipelago, Indian Ocean) Revue. Écologie. (Terre Vie), $60: 325-332$

6. Brun, C. (2007). Archéophytes et néophytes pour une nouvelle détermination des marqueurs polliniques de l'anthropisation. Le cas des milieux cultivés et rudéraux de Franche-Comté. Thèse de Doctorat de l'Université de Franche-Comté (France), 430 p.

7. Carrière, M., S., Randrianasolo, E., \& Hennenfent, J. (2008). « Aires protégées et lutte contre les bioinvasions : des objectifs antagonistes ? Le cas de Psidium cattleianum Sabine (Myrtaceae) autour du parc national de Ranomafana à Madagascar. » VertigO - la revue électronique en sciences de l'environnement [En ligne], Volume 8 Numéro 1 | avril 2008, mis en ligne le 12 avril 2008, consulté le 06 Septembre 2018. URL : http://journals.openedition.org/vertigo/1918 ; DOI : 10.4000/vertigo.1918.

8. Chatelain, C., Gautier, L., \& Spichiger R. (2001). Application du SIG ivoire à la distribution potentielle des espèces en fonction des facteurs écologiques, Systematics and Geography of Plants 71 : 313-326. 
9. De Foresta, H. (1995). Systèmes de culture adventices envahissantes et fertilité du milieu : le cas de Chromolaena odorata : InPichot J., Sibelet N., Lacoevilhe J.J. (eds) Fertilité du milieu et stratégies paysannes sous les tropiques humides. CIRAD, Ministère de la Coopération, Montpellier (FRA), Paris 236-244.

10. De Koning, J. (1983). La forêt du Banco. Thèse de Doctorat, Université de Wageningen, Pays-Bas, 1500 p.

11. Dietz, H., \& Edwards, P., J. (2006). "Recognition that causal processes change during plant invasion helps explain conflicts in evidence", Ecology, 87 (6): 1359-67.

12. Dumitrascu, M., Kucsicsa, G., Grigorescu, I., Dragota, C-S., \& Nastase, M. (2012). Invasive terrestrial plant species in the Romanian protected areas. Case study: Follopia japonica in the Maramures Mountains Natural Park, forum geographic 9 (1): 45-53.

13. Eldin, M. (1971). Le climat de la Côte d'Ivoire. In : Le milieu naturel de Côte d'Ivoire, Mémoires ORSTOM, 50, Paris (France), 73-108.

14. F.A.O, (2006). World reference base for soil resources. Aframework for international classification, correlation and communication. World soil resources reports 103, 145p.

15. Ferraris, J. ; Chevillotte, H., Meyer, J-Y., Mellado, T., Florence, J., Emmanuelli, E., Habert, E., Galzin R. (2006). Suivi des phénomènes bio-invasifs et de restauration de la biodiversité dans des aires protégées marines et terrestres : l'exemple de Moorea en Polynésie française. Paris: IRD, 32p.

16. Foxcroft, L., C., Henderson, L., Nichols, G., R., \& Martin B., W. (2003). A revised list of alien plants for the Kruger National Park, Koedoe 46 (2), 21-44.

17. Foxcroft, L., C., Richardson, D., M., Pysek, P., \& Genovesi, P. (2013). Plant invasion in protected areas: outlining the issue and creating the links. Pp 3-18. In: L.C. Foxcroft, P. Pysek, D.M. Richardson \& P. Genovesi (eds.). Plant invasions in protected areas: patterns, problems and challenges, Invading Nature Springer, Dordrecht.

18. Foxcroft, L., C., Pysek, Richardson, D., M., Genovesi, P., \& MacFadyen, S. (2016). Plant invasion science in protected areas: progress and priorities Biological Invasions DOI 10.1007/s10530016-1367-z.

19. Gautier, L., Aké Assi, L., Chatelain, C., \& Spichiger, R. (1999). African Plants: Biodiversity Taxonomy and uses, chapter Ivoire: a geographic information system for biodiversity managment in Ivoiry Coast, Royal Botanic Garden,183-194.

20. Gueyé, M., Cissé ; M., T., Ndoye, F., \& Noba, k. (2015). Dynamique invasive de Mimosa pigra dans un contexte de variabilité du regimbe 
hydrologique au Sénégal, Journal of Animal \&Plant Sciences, .26, (1) : 4023-4035.

21. Hauhouot, A. (2002). Développement, Aménagement, Régionalisation en Côte d'Ivoire. EDUCI, 364 p.

22. Haury, J., \& Damien, J-P. (2012). Les invasions biologiques dans le Parc naturel régional de Brière : présentation d'une recherche- action, Sciences Eaux \& Territoires 6, 26-33.

23. Hulme, P., E. (2016). Climate change and biological invasions: evidence, expectation and response option, Biological Revue. doi: 101111/brv.12282.

24. Hulme, P., E., Pyšek, P., Nentwig, W., \& Vilà, M (2009). Will threat of biological invasions unite the European Union? Science 324, 40-41.

25. Jourdan, H., Chazeau, J. 1(999). Les fourmis comme bio-indicateurs : l'exemple de la myrmécofaune néo-calédonienne. Actes Colloque Insectes Sociaux (12) : 165-170.

26. Lauginie, F. (1996). Propositions pour l'avenir des parcs nationaux et réserves naturelles de Côte d'Ivoire. DDC/MINAGRA/WWF, Abidjan, 86p.

27. Lauginie, F. (2007). Conservation de la nature et aires protégées en Côte d'Ivoire, NEI / Hachette et Afrique Nature, Abidjan, xx +668p.

28. Leavold, V., Llyod, L., \& Lepetit, J. (2007). Development of case studies on the economic impacts of invasive species in Africa. Technical report, Global Invasive Species Program, 100p.

29. Lebrun, J., P. \& Stork, A., L. (1991). Énumération des plantes à fleurs d'Afrique tropicale, Ville de Genève, Editions des Conservatoire et Jardin botaniques, $249 \mathrm{p}$.

30. Lebrun, J., P. \& Stork A. L. (1997). Énumération des plantes à fleurs d'Afrique tropicale : 4 Gamopétales : Clethraceae à Lamiaceae, 712 p.

31. Levine, J., M. (2008). Biological invasions. Current Biology, 18 : 57 60. Lowe S., Browne M., Boudjelas, S., \& De Poorter, M. (2000). 100 of the most world's worst invasive alien species. A selective from global invasive database. Technical report, The Invasive Species Specialist Group (ISSG), Species Survival Commission (SSC), World Conservation Union (IUCN), 11 p.

32. MacDonald, I., A., W., Loope, L., L, Usher, M., B., Hamann, O. (1989). Wildlife conservation and the invasion of nature reserve by introduced spcies: a global perspective. In invasion: A global perspective. In "Biological invasions: A global perspective", Drake J.A., Mooney, H.A., Di Castri, F., Groves, R.H. Kruger, K.J., Rejmanek M. Williamson, M. (Eds), John Wiley \& Sons, Chichester: 215-256. 
33. McNeely, J., A., Mooney, H., A., Neville, L., E., Schei, P., \& Waage, J., K. (Eds) (2001). A Global Strategy on Invasive Alien Species. IUCN Gland \& Cambridge in collaboration with the Global Invasive Species Programme. 50p.

34. Mèmèl, J., D., Karamoko, M., Otchoumou, A., \& Kouassi, D., K. (2011). Abondance, taille et mortalité des escargots terrestres du Parc National du Banco (Côte d'Ivoire) : effet de la composition granulométrique et chimique du sol, Livestock Research for Rural Development 23(9) 6p.

35. Neuba, D., F., R., Malan, D., F., Koné, M., \& Kouadio Y., L. (2014). Inventaire préliminaire des plantes envahissantes de la Côte d'Ivoire, Journal of Animal \& Plant Sciences 22(2), 3439-3445.

36. Ngueguim, J., R., Zapfack, L., Youmbi, E., Bernard Riera, B., Onana, J., Foahom, B., \& Makombu, J., G. (2010). Diversité floristique sous canopée en plantation forestière de Mangombe-Edea (Cameroun), Biotechnology, Agronomy, Society and Environment, 14(1) : 167-176.

37. N'Guessan, A., E., \&. Kassi N., J. (2018). Analyse De La Diversité Floristique De La Forêt Classée D'agbo I (Côte d'Ivoire). European Scientific Journal, 14(9) :346. https://doi.org/10.19044/esj.2018.v14n9p346.

38. Noba, K., Bassene, C., Ngom, A., Gueye, M., Camara, A., A., Kane., M., Ndoye, F., Dieng, B., Rmballo, R., Ba, N., Bodian, M., Y., Sane, S., Diop, D., Konta, S., Kane, A., Mbaye, M., S., \& Ba, A., T. (2017). Invasive Plants of West Africa: Concepts, Overviews and Sustainable Management. Adv Recycling Waste Manag 2 121. DOI : 10.4172/2475-7675.1000121.

39. Nyakabwa, M. (1981). Flore et végétations rudérales de la zone Makiso à Kisangani (Haut-Zaire) étude floristique, phytosociologique et écologique, DEA, Université de Kisangani République Démocratie du Congo, faculté des sciences, 216p.

40. Nzengue, E., Sambou, B., Thiam, A., Noba, K., Sambou, Y., \& Mavoungou, J-F. (2016). Prolifération de Cissus quadrangularis L. dans le Parc National des Iles-de-la-Madeleine, Sénégal, VertigO - la revue électronique en sciences de l'environnement [En ligne], Regards / Terrain, mis en ligne le 04 avril 2016, consulté le 25 avril 2018. URL : http://journals.openedition.org/vertigo/16880 ;

DOI : 10.4000/vertigo. 16880.

41. Osseni, A., A., Tohozin, C., A., B., Toko -Mouhamadou, I., \& Sinsin B. (2014). Contribution des SIG dans l'analyse floristique des espaces verts dans la ville de Porto-Novo au Bénin, Revue Ivoirienne des Sciences et Technologie, 23, 103-121. 
42. Peel, M., C., Finlayson, B., L., \& McMahon, T., A. (2007)."Updated world map of the koppen- Geiger climate classification", Hydrology and Earth System Sciences, 11(5): 1633-1644.

43. Perraud, A. (1971) Les sols. In : Le milieu naturel de la Côte d'Ivoire. Mémoire ORSTOM, 50, Paris (France), 157-263.

44. Perrings, C. (2005). The socioeconomic link between invasives alien species and poverty. Technical report, Global Invasive Species Program, $36 \mathrm{p}$.

45. Pimentel, D., L., Lach, K., Zaniga, \&. Morrison D. (2000). Environnemental and economic cost of nomardegenous species in the United States, Bioscience, 50 : 53-65.

46. Saad, L., Jacquemart, A-M., Gawoy, V., Vampary, V., \& Vervoort, A. (2009). Les plantes envahissantes en Belgique ont-elles des impacts? In : Parcs et réserves : revue trimestrielle d\&amp ; apos, Andenne et Gaume 64(4), 10-17.

47. Roux, R. (2011). Les espèces animales invasives dans le département de la Sarthe. Master 2 Professionnel « Gestion de l'environnement », Université Bordeaux IV (France), 173p.

48. Sako, N., \& Beltrand, G. (2014). «Dynamiques spatiales récentes du Parc National du Banco (PNB) et stratégie de gestion communautaire durable de ses ressources forestières (District d'Abidjan en Côte d'Ivoire) »Echo Géo [En ligne], 30 | 2014, mis en ligne le 17 septembre 2014, consulté le 04 Août 2018. URL : http://echogeo.revues.org/13904 ; DOI :10.4000/echogeo.13904.

49. Sako, N., Beltrando, G., Atta, K., L., N'da, D., H., \& Brou, T. (2013). «Dynamique forestière et pression urbaine dans le Parc national du Banco (Abidjan, Côte d'Ivoire) » VertigO - la revue électronique en sciences de l'environnement [En ligne], Volume 13Numéro 2 | septembre 2013, mis en ligne le 06 octobre 2013, consulté le 16 août 2018. URL: http://vertigo.revues.org/14127; DOI: 10.4000/ vertigo. 14127.

50. Salonen, V. (1994). Growth and reproduction of Rubus saxatilis L. in relation to availability of light, Acta OEcologica, 15: 485-493.

51. Selmi, W. (2016). Evaluation des services écosystémiques rendus par les arbres urbains, étude de l'effet des arbres sur l'environnement urbain, résultats de l'application du modèle i- Tree Eco à la ville de Strabourg. (Rapport Technique) Laboratoire Image, Ville, Environnement à Strabourg, 20p.

52. Selvi, F., Carrari, E., \& Coppi, A. (2016). Impact of invasion on the taxonomic and phylogenetic diversity of a relict Mediterranean forest ecosystem, Forest Ecology Management, 367: 1-11. 
53. Simberloff, D. (2016). Jacques Tassin : La grande invasion : Qui a peur des espèces invasives ? Biological Invasions 18: 295-297. Book review. doi: 10.1007/s10530-015-0994-0.

54. Thiombiano, N., Ouedraogo, R., L., Belem, M., \& Guinko, S. (2009). Dynamique de l'évolution et impact d'une plante envahissante au Burkina Faso Hyptis Suaveolens (L.) Poit. Annal de Université du Lomé (Togo), série Sciences, Tome XVIII : 97-115.

55. Tia, L., \& Dago, D. (2015). Morcellement d'une aire protégée en agglomération urbaine : le cas du Parc National du Banco (Côte d'Ivoire). Cahiers de géographie du Québec, 59 (168), 349- 376. Doi :10.7202/1037254ar.

56. Tiébré, M-S., Vanderhoeven, S., Saad, L., \& Mathy, G. (2007). Hybridization and sexual reproduction in the invasive Alien Fallopia (Polygonaceae) complex in Belgium, Annals of Botany 99, 193-203.

57. Tiébré, M-S., Saad, L., \& Mahy, G. (2008). Landscape dynamics and habitat selection by alien invasive Fallopia (Polygonacea) in Belgium, Biodiversity and Conseveation. 17 (10): 2357-2370.

58. Tiébré, M-S., Vroh Bi, T., A., Kouamé, D., N’Da, K., D., \& Adou, Y., C-Y. (2014). Stratégies et potentiel d'invasion des massifs forestiers par Hopea odorata Roxb. (Dipterocarpaceae) : cas du Parc National du Banco en Côte d'Ivoire, International Journal of Biological and Chemical Science 8 (2), 666-679.

59. Tiébré, M-S., Vroh Bi, T., A., Kouamé, D., N’Da, K., D., \& Adou Y., C-Y. (2015). Effets d'un arbre exotique envahissant Hopea odorata Roxb. (Dipterocarpaceae) sur la diversité floristique et le stockage de carbone du Parc National du Banco en Côte d'Ivoire, International Journal of Innovation and Applied Studies, 10 (1), 207-216.

60. Tiébré, M-S., \& Gouli Gnanazan Z., R. (2018). Impact of Chromolaena odorata (L.) R.M. King \& H. Rob. (Asteraceae) on the floristic composition and the physico-chemical properties of the soil of a coastal relict forest, International Journal of Innovation and Applied Studies, 24 (2): 773-788.

61. Tyser, R., W., \& Worley, C., A. (1992). Alien flora in Usha K, Prasad R, Muniappan P, Ferrar JP, Aeschliman, H. De Foresta (1996) Répartition, écologie et gestion de Chromolaena odorata. Comptes Rendus du Troisième Atelier International sur Chromolaena odorata Abidjan, Côte d'Ivoire, November 1993. Agricultural Experiment Station University of Guam, Mangilao, GUAM, USA Publication $\mathbf{n}^{\circ}$ 202, 213p.

62. Schatz, T., J. (2001). The effect of cutting on the survival Mimosa pigra and its application to the use of blade ploughing as a control method, Plant Protection Quarterly 16(2). 
63. Scherer, B. (1984). Biostatistique, Édition Morin Gaëtan, 850p.

64. Strasberg, D. (1995). Processus d'invasion par les plantes introduites à la Réunion et dynamique de la végétation sur les coulées volcaniques. Ecologie 12(3) :169-180.

65. Uchida, T., Xue, J., H., Hayasaka, D., Arase, T., Haller, T., W., \& Gettys L., A. (2014). The relation between road crack vegetation and plant biodiversity in urban landscape, International Journal of Geomate6 (2), 885-891.

66. Udo, N., Darrot, C., Tarayre, M., \& Atlan, A. (2016) « Histoire humaine et naturelle d'une invasion biologique ", Revue d'ethnoécologie [En ligne], 9 | 2016, mis en ligne le 01 juillet 2016, consulté le 01/11/2018. URL : http://ethnoecologie.revues.org/2724 ; DOI : 10.4000/ethnoecologie.2724.

67. UICN. (2000). Lignes directrices de l'UICN pour la prévention de la perte de la diversité biologique causée par les espèces exotiques envahissantes. UICN, Commission de sauvegarde des espèces, 25 p.

68. UICN/ PACO. (2013). Plantes invasives affectant les aires protégées de l'Afrique de l'Ouest : gestion pour la réduction des risqué pour la biodiversité. UICN/PACO : Ouagadougou, Burkina- Fasso. 52p.

69. Usher, M. (1988). Biological invasions of nature reserve a search for generalizations, Biological Conservation 44: 119-135.

70. Walden, D., VanDam, R., Finlayson, M., Stors, M., Lowry, J., Kriticos, D. (2004). A risk Assessment of the tropical wetlands weed Mimosa pigra in northern Australia, supervising Scientist, report 177.

71. Williams, N., S., Hahs, A., K., \& Vesk, P., A. (2015). Urbanization, plant traits and the composition of urban floras. Perspectives in Plant Ecology, Evolution and Systematics, 17(1):78-86. 